\title{
How Many Angels Can be in the Same Place at the Same Time? A Defense of Mereological Universalism
}

\author{
A. J. COTNOIR \\ University of St Andrews, St Andrews, KY16 9AR, United Kingdom \\ ac117@st-andrews.ac.uk
}

I reply to Hawthorne and Uzquiano's (2011) arguments for the incompatibility between mereological universalism and plenitudinous co-location. I argue that a mereology in which antisymmetry for parthood fails is independently motivated, and allows for both universalism and plenitudinous co-location. There can be as many angels in a place as there are cardinalities.

Supposing that angels can be located at a (zero-dimensional) point in space, and additionally that they can be co-located, the question naturally arises: how many such things can be colocated at a point in space? Where alephs are cardinals, here are two possible answers.

Indefinite Extensibility There could not be so many angels as to exceed each and every aleph, but for each ordinal $\alpha$ there could be at least $\aleph_{\alpha}$-many angels located at a point.

Plenitude The could be at least as many angels as there are alephs.

Hawthorne and Uzquiano (2011) object to PLENITUde by showing that it incompatible with mereological universalism - the view that for every plurality $x x$, there exists of fusion of $x x$. (They also show how the weaker answer of INDEFINITE EXTENSIBILITY is incompatible with Lewis's (1986) modal realism and Williamson's (2013) necessitism.) Instead of their argument constituting a reductio against PLENITUDE (or against INDEFINITE EXTENSIBILITY for modal realists/necessitists), one might rather conceive of it as a reductio of mereological universalism.

1. If mereological universalism is true, then it is necessarily true.

2. If mereological universalism is necessarily true, then PLENITUDE is false.

3. But, Plenitude is true.

4. So, mereological universalism is false. 
One might reject (1) a la Cameron 2007 and Parsons 2013. ${ }^{1}$ Or, one might reject (3) as apparently do Hawthorne and Uzquiano. Finally, one might reject (2) and Hawthorne and Uzquiano's case for it. In this article I argue against (2) by claiming that the mereological universalist who believes in co-location has a straightforward and independently-motivated reply.

\section{Videtur quod non}

It would seem that there cannot be as many angels as there are alephs. For consider the following widely accepted principle connected to the philosophical conception of a set.

Limitation of Size A plurality forms a set iff they are not in one-one correspondence with the entire universe of all objects.

This principle entails that there is exactly one cardinality that is not characterised by the alephs, and that is the size of the universe. To see why, note that (on pain of the Burali-Forti paradox) the ordinals do not form a set. So, by LIMITATION OF SIZE the ordinals must be in a one-one correspondence with the entire universe. But since the aleph series can be put into a one-toone correspondence with the ordinals, we know that the size of the alephs is the only size not characterised by them.

Say that a plurality $x x$ is disperse iff every sub-plurality $y y$ among the $x x$ has a distinct mereological fusion. We also can show, following Hawthorne and Uzquiano 2011, p. 61-2, by a generalization of Cantor's diagonal argument, that any non-singular plurality has more sub-pluralities than members. What follows, then, is a particular mereological result:

Mereological Result if a non-singular plurality is disperse, there are more fusions of its subpluralities that there are members of it.

Hawthorne and Uzquiano's (2011, p. 62) argument is as follows: suppose we have a non-singular disperse plurality. Then it has more sub-pluralities than members. By mereological universalism, every sub-plurality has a fusion. By disperseness, distinct sub-pluralities have distinct fusions. Thus, it has more fusions of its sub-pluralities that it has members.

Notice that this mereological result rests crucially on the assumption of mereological universalism: once we assume that every sub-plurality of a disperse plurality has a fusion, then the plurality has more fusions than members.

${ }^{1}$ Although, there are some costs to this approach. A mereological universalist who rejected (1) would need to make apparently ad hoc restrictions on the contingency of composition: while it would be contingent whether finitely many co-located objects composed, it would have to be necessary that infinitely many co-located objects do not compose, on pain of contradiction, as Hawthorne and Uzquiano show. (Thanks to an anonymous referee for this point.) 
At last, we come to the argument.

Our [mereological] result tells us that there are strictly more fusions of angels than there are angels. Limitation of size tells us that the size of the [angels] is at most the size of the alephs. [...] Given Plenitude, we are forced to conclude that the size of the angels matches the size of the actual alephs. But now, by our mereological result, we must conclude that there are strictly more [fusions of angels] than there are alephs, which contradicts limitation of size. (Hawthorne and Uzquiano, 2011, p. 66-7)

It would seem, then, that we cannot accept both PLENITUDE and mereological universalism.

\section{Sed contra}

To the contrary, Hawthorne and Uzquiano suggest the possibility of a non-standard mereology, when discussing their objection to Williamson's necessitism:

Suppose we adopt a mereology that - as against classical extensional mereology - abandons the presumption that parthood is antisymmetric [...] It now becomes possible to think of the possibly (but not actually) concrete objects, not as forming a disperse plurality, but as parts of each other, forming an entangled unity. When an object becomes concrete it breaks off from - that is, becomes mereologically discrete from those entangled entities, and when it ceases to be concrete, it returns to - that is, becomes mereologically reconciled to - those entities. While this is not perhaps a full vindication of Plotinus' doctrine of a return to the One - carried into scholastic philosophy by the early Church Fathers - it is perhaps as close to a vindication as sober analytic metaphysics can provide. (Hawthorne and Uzquiano, 2011, p. 73)

While Williamson's necessitism does utilise the concrete/abstract distinction in crucial and controversial ways, he does nothing so far removed from sober analytic metaphysics as this. This sort of failure of antisymmetry seems under-motivated, and so the rejection of standard mereology seems here to be ad hoc.

\section{Respondeo dicendum}

I reply saying that one does not have to appeal to any strange doctrines like the 'return to the One' to defend mereological universalism. Indeed, the failure of antisymmetry is much better moti- 
vated in the context of mereological universalism that allows for the possibility of co-location. That is, there are independent reasons why co-located angels should be thought to constitute counterexamples to antisymmetry.

To see this, we begin with a single binary primitive of mereological overlap $\circ$ and the following definitions. ${ }^{2}$

Parthood $x \leq y:=\forall z(z \circ x \rightarrow z \circ y)$

Proper Parthood $x<y:=x \leq y \& y \not \leq x$

Non-identical Parthood $x \lessgtr y:=x \leq y \& x \neq y$

Fusions $F(y, x x):=\forall z(z \circ y \leftrightarrow z \circ x x)$

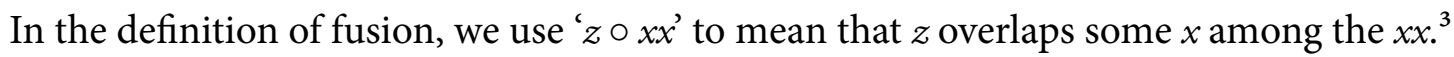

The definition of parthood allows us to prove reflexivity and transitivity of $\leq$; but we do not allow anti-symmetry of $\leq$ which is equivalent to $\forall z((z \circ x \leftrightarrow z \circ y) \rightarrow x=y)$, an extensionality principle of overlap. Thus, we might have distinct objects $a \neq b$ such that $a \leq b$ and $b \leq a$. As a consequence, we need to be clear about the relevant notion of proper part that we are using. On one conception, $x$ is a proper part of $y$ whenever $x$ is non-mutual part of $y$. By the definition of parthood in terms of overlap, we thus have that if $x<y$ then there's a $z$ which overlaps $x$ but doesn't overlap $y$. This is a form of weak supplementation. By contrast, there is another conception of proper part - that of a non-identical part, expressed by ' $\leq$ ' - according to which we are not guaranteed supplementation.

In any case, we have two axioms of our mereology:

Axiom $1 \quad \forall x \forall y(x \circ y \leftrightarrow \exists z(z \leq x$ \& $z \leq y))$

Axiom $2 \forall x x \exists y F(y, x x)$

The first axiom is simply an intuitively obvious truth about overlap. The second axiom is tantamount to mereological universalism.

But this mereology is very well-suited to allowing co-location. Indeed, it may well be the only co-locational mereology compatible with the following assumption about locations. Where an object is weakly located at a region iff the region is not completely free of it,

\footnotetext{
${ }^{2}$ Note that we are using $\circ$ to correspond to mereological overlap - not mere spatial overlap. Alternatively, we could have chosen mereological disjointness $?$ as our primitive, since they are interdefinable as the negations of each other.

${ }^{3}$ These are fusions in the style of Goodman 1951 and Simons 1987, rather than the definition of fusions used in Tarski 1929 and Lewis 1991. Although this mereology could have been axiomatized using the Tarskian definition, too (see Cotnoir 2014).
} 
Harmony if $x$ is weakly located at every region in which $y$ is weakly located, then anything that overlaps $x$ overlaps $y .{ }^{4}$

On this view, co-located objects $x$ and $y$ are mutual parts: $x \leq y \& y \leq x .{ }^{5}$ By the definition of $\leq$, this is equivalent to: $\forall z(z \circ x \leftrightarrow z \circ y)$; that is, two objects are co-located whenever they overlap the same objects.

\section{Ad primum ergo dicendum}

Therefore to the first objection based on the LIMITATION OF SIZE, we can see that on this mereological conception of co-located $x x$ and any $x_{i}$ among the $x x$, we have it that $F\left(x_{i}, x x\right)$; after all, for co-located $x x, \forall z\left(z \circ x_{i} \leftrightarrow z \circ x x\right)$. In the case at hand, each individual angel counts as a fusion of the angels. Hence according to our co-location mereology, the angels are not a disperse plurality. So the Limitation OF SIZE argument fails. ${ }^{6}$ We do need some assurance that the only fusions of $x x$ that exist are identical to some $x_{i}$ among the $x x$. In other words, we want to be sure that the angels together with their fusions do not force there to be strictly more things than there are alephs.

There is, however, a straightforward way of showing why the number of fusions of angels is just the same as the number of angels themselves. We merely need appeal to a single plausible principle about locations of fusions:

Inheritance If $y$ is the fusion of $x x$, then for all regions $r, y$ is weakly located at $r$ iff some $x$ among $x x$ is weakly located at $r$.

This principle entails that if none of the $x x$ are located in a region, then neither is their fusion. That is, a whole inherits the locations of its parts. We can then argue as follows. Let the $x x$ be the

${ }^{4}$ Alternatively: the exact location of $x$ is a subregion of the exact location of $y$ iff $x \leq y-$ the harmony principle called $(1 \rho)$ in Uzquiano 2011 , p. 204.

${ }^{5}$ It might seem odd that on this view spatial coincidence would entail mereological overlap; why couldn't two distinct things occupy the same place without being mereologically related in any way? The view is considered ( $\mathrm{p}$. 117) and eventually rejected (Ch. 6) in Simons 1987. I do not have the space to defend the view here, but here are two preliminary reasons in favour of the view. The first is that HARMONY follows from a more general principle to the effect that the exact location of a fusion is inherited from the exact locations of its parts (see Uzquiano 2011, p. 205, and Fn. 17). A second reason is that it allows for a purely spatial approach to mereology in the sense of Markosian 2014. In other words, accepting this harmony principle would allow a reduction of mereology to purely topological predicates and relations (see Casati and Varzi 1999, Sect. 4.4) without requiring that co-located objects are identical.

${ }^{6}$ One might object that while this response preserves the letter of mereological universalism, it gives up the spirit of universalism since the fusion of co-located objects need not be some further thing. Just what counts as mereological universalism is somewhat less clear in the context where co-location is allowed, and indeed is made more complicated by a non-extensional mereological theory as required here. For more discussion showing that a related view satisfies every possible plausible definition of universalism, see Cotnoir 2014 . 
plurality of all things exactly located at a point $r{ }^{7}$ By LIMITATION OF SIZE, the $x x$ can be at most as many as the alephs. Now, let $F(y, x x)$. Since fusions are upper bounds of the things fused, $x x$ are all parts of $y$. Hence, by INHERITANCE, $y$ is weakly located at $r$ and only $r$; that is, $y$ is exactly located at $r$. Hence $y$ is one of the $x x$. As a result we can rest assured that the cardinality of angels does not exceed the cardinality of the alephs. ${ }^{8}$

\section{References}

Cameron, R. P., 2007: The contingency of composition. Philosophical Studies, 136, 99-121.

Casati, R. and A. C. Varzi, 1999: Parts and Places: The Structures of Spatial Representation. MIT Press, Cambridge (MA).

Cotnoir, A. J., 2014: Does universalism entail extensionalism? Noûs, EarlyView.

Goodman, N., 1951: The Structure of Appearance. Harvard University Press, Cambridge, MA.

Hawthorne, J. and G. Uzquiano, 2011: How many angels can dance on the point of a needle? Mind, 120(478), 53-81.

Lewis, D. K., 1986: On the Plurality of Worlds. Blackwell.

Lewis, D. K., 1991: Parts of Classes. Basil Blackwell, Oxford.

Markosian, N., 2014: A spatial approach to mereology. In Mereology and Location, Kleinscmidt, S., editor. Oxford University Press, 69-90.

Parsons, J., 2013: Conceptual conservatism and contingent composition. Inquiry, 56(4), 327339.

Simons, P. M., 1987: Parts: A Study In Ontology. Clarendon, Oxford.

Tarski, A., 1929: Les fondements de la géométrie des corps. Ksiega Pamiatkowa Pierwszkego Polskiego Zjazdu Matematycznego, 7, 29-33. English translation by J. H. Woodger in Tarski 1956.

Tarski, A., 1956: Logic, Semantics, Metamathematics. Hackett, Indianapolis. J. Corcoran (ed.).

Uzquiano, G., 2011: Mereological harmony. In Oxford Studies in Metaphysics, Bennett, K. and Zimmerman, D., editors, volume 6. Oxford University Press, 199-224.

Williamson, T., 2013: Modal logic as metaphysics. Oxford University Press.

\footnotetext{
${ }^{7} \mathrm{I}$ am here ignoring the additional complications that might result from allowing multi-location.

${ }^{8}$ Thanks to Franz Berto, John Hawthorne, Bruno Jacinto, Carrie Ichikawa Jenkins, Martin Lipman, Toby Meadows, Stephen Read, Gabriel Uzquiano, the ARCHÉ Metaphysics Research Group, and seminar participants in the Northern Institute of Philosophy at the University of Aberdeen for discussion.
} 